\title{
TAMAÑO ÓPTIMO DE UNIDAD EXPERIMENTAL PARA ENSAYOS EN ARROZ BAJO EL IMPACTO DE BIO-AGENTES QUE PUEDEN TENER INFLUENCIA SIGNIFICATIVA SOBRE LA VARIABILIDAD DEL SUELO EN BAGACES, GUANACASTE
}

\section{OPTIMUM PLOT SIZE FOR RICE TRIALS UNDER THE IMPACT OF BIO-AGENTS WITH A SIGNIFICANT INFLUENCE ON SOIL VARIABILITY IN BAGACES, GUANACASTE, COSTA RICA}

Juan Ramón Navarro-Flores ${ }^{1}$ Jorge Claudio Vargas-Rojas ${ }^{2}$

\begin{tabular}{|l|l}
\hline Recibido: 05.04.19 & Aprobado: 28.11.19
\end{tabular}

DOI: $10.15517 /$ isucr.v20i42.41904

\section{Resumen:}

Se realizó un estudio con el objetivo de determinar si la adición de productos a base de microorganismos podrían aumentar, por medio de su dinámica natural y sus interaccione con las poblaciones de microflora y microfauna nativas, la variabilidad del suelo y por ende el tamaño óptimo de la unidad experimental para ensayos en arroz en el cantón de Bagaces, Guanacaste, Costa Rica. Este ensayo se realizó en la misma finca donde se realizó el trabajo original en que se definió el tamaño óptimo de parcela para ensayos de arroz en la zona (Vargas y Navarro 2014). Se sembró cuatro ensayos de uniformidad, uno por cada producto utilizado; Trichoderma, Bioactivado, Nitrobacter y Micro Plus. Los datos obtenidos por este medio fueron empleados para aplicar el método de curvatura máxima. El punto de curvatura máxima -determinado por medio de la derivada parcial de la ecuación de regresión que relaciona el tamaño de la unidad experimental (X) con su correspondiente coeficiente de variación (Y)- para ningún producto superó el ámbito definido por Vargas y Navarro (2014) de $15 \mathrm{~m}^{2}$ a $20 \mathrm{~m}^{2}$. Los índices de heterogeneidad obtenidos en ningún caso superaron el valor de 0,83 (el ámbito de la escala para esta medida va de 0 a 1 , donde 0 indica total homogeneidad y 1 total heterogeneidad) (Vargas y Navarro 2014) lo que demuestra que en una misma finca se pueden encontrar suelos muy diversos. Por último, el análisis de variancia mostró diferencia entre la producción obtenida con cada uno de los productos; con el producto Bioactivado se obtuvo una producción de $17 \mathrm{tm} / \mathrm{ha}$ que triplica la producción normal de la finca.

\footnotetext{
${ }^{1}$ Docente. Escuela de Agronomía, Sede Rodrigo Facio, Universidad de Costa Rica; correo electrónico: juan.navarro@ucr.ac.cr

2 Docente. Escuela de Agronomía, Sede Guanacaste, Universidad de Costa Rica; correo electrónico: jorgeclaudio.vargas@ucr.ac.cr
} 
Palabras clave: Microorganismos; tamaño de unidad experimental; arroz; heterogeneidad del suelo; producción de grano.

\begin{abstract}
:
This study was conducted in order to determine whether adding products based on microorganisms could increase, through their natural dynamics and their interaction with native microflora and microfauna populations, the variability of the soil and, therefore, the optimum plot size for rice trials in Bagaces, Guanacaste, Costa Rica. This trial was conducted at the same farm where the optimum plot size was defined for rice trials in the area (Vargas \& Navarro 2014). Four uniformity trials were planted, one for each product used: Trichoderma, Bioactivado, Nitrobacter, and Micro Plus. The data obtained was used to apply the maximum curvature method. The point of maximum curvature was determined using the partial derivative of the regression equation, which relates the plot size $(\mathrm{X})$ with the corresponding variation coefficient $(\mathrm{Y})$. None of the product's point of maximum curvature exceeded the scope defined by Vargas \& Navarro (2014) from $15 \mathrm{~m}^{2}$ to 20 $\mathrm{m}^{2}$. None of the indices of heterogeneity obtained exceeded the 0.83 value (the scope of the scale for this measure ranges from 0 to 1 , where 0 indicates total homogeneity and 1 total heterogeneity) (Vargas \& Navarro 2014), which shows that very diverse soils can be found in the same farm. Finally, the variance analysis showed differences in the production obtained with each product. A $17 \mathrm{tm} /$ ha production was obtained with Bioactivado, which triples the normal production of the farm.
\end{abstract}

Keywords: Microorganisms; plot size; rice; soil heterogeneity; grain production.

Introducción

El mejoramiento genético con el fin de aumentar la producción o cualquier otra característica deseable en un cultivo debe basarse en la optimización del dinero invertido: esto es: información confiable, para que tanto las empresas como los agricultores puedan acogerlas con seguridad (Barrientos 1981). Chandler (1984) se refiere a los elementos esenciales de un programa de investigación en arroz, donde señala la utilidad de ciertos métodos y prácticas dentro de los cuales se encuentra la determinación de un tamaño óptimo de unidad experimental.

Para determinar el tamaño óptimo de unidad experimental existen métodos estadísticos muy variados, la mayoría parten de un ensayo blanco o ensayo de uniformidad (Rodríguez et al. 1993; Custódio 2010; Santos et al. 2012), si bien existen otros métodos, éste es el más preciso, con el inconveniente de que requiere de una inversión considerable de tiempo y recursos (Álvarez et al. 1986).

Los ensayos blancos son extensiones relativamente grandes que son tratadas en toda su superficie uniformemente en cuanto a todas las labores culturales relacionadas con el cultivo. A la hora de la cosecha se subdivide en parcelas pequeñas (unidades básicas), y luego estas unidades 
básicas se agrupan de manera que formen parcelas de diferentes formas y tamaños. Para determinar la producción de estas agrupaciones se suma el rendimiento individual de las unidades básicas que se juntan para formar las unidades secundarias. La comparación de cada tipo de parcela se hace recurriendo al error probable, error típico o al coeficiente de variación (Rosselló y Fernández 1986; Kavitha 2010).

El método más utilizado para relacionar el tamaño de las unidades secundarias (Y) con el coeficiente de variación $(\mathrm{X})$ es el método denominado "curvatura máxima"; esto produce una figura de dispersión que puede ser representada por medio de una función matemática 87u’p-que a su vez se puede derivar para obtener el punto de curvatura máxima de la función (Escobar 1981). La medida de la heterogeneidad (Smith 1938) del suelo es otro subproducto de los ensayos blancos (Gomez y Gomez 1984), ya que es precisamente la variabilidad del suelo la que determina el tamaño adecuado de unidad experimental. Esta variabilidad de los suelos puede deberse a factores físicos, químicos o biológicos y que, junto con otros factores como la genética del cultivo y las características del sitio, introducen variación en variables como la producción de un cultivo y que se conoce como error experimental (Box et al. 2008).

Los agentes biocontroladores se emplean cada vez más en agroecosistemas de todo tipo, el arroz no es una excepción. Estos agentes actúan incorporándose en la rizosfera del cultivo donde adicionan su dinámica particular a la dinámica de otras poblaciones de microflora y microfauna pre-existentes en el suelo; esta adición junto con la dinámica propia del cultivo puede estar creando un sistema más variable y por ende los cálculos con que se cuenta actualmente de tamaño de unidad experimental se podrían estar quedando cortos con el consecuente daño a la confiabilidad de los resultados obtenidos en la experimentación con arroz. El objetivo general de este trabajo fue determinar si la adición de agentes biológicos al cultivo del arroz implica un aumento significativo de la heterogeneidad del suelo y por ende un aumento en el tamaño de la unidad experimental recomendada de $20 \mathrm{~m}^{2}$

\section{Metodología}

El ensayo se llevó a cabo durante los meses de junio a noviembre del año 2016, en la Hacienda Mojica, situada en el cantón de Bagaces, provincia de Guanacaste, la cual se encuentra a 80 msnm, con una precipitación que oscila entre 1500 a $2500 \mathrm{ml} / \mathrm{año} \mathrm{y} \mathrm{temperatura} \mathrm{promedio}$ 
anual de $29^{\circ} \mathrm{C}$. La hacienda se encuentra en la zona de vida "bosque tropical seco" (Bolaños et al. 2005) y su actividad económica principal es la producción de arroz.

Los tratamientos empleados fueron (Primuslabs 2016): a) Micro Plus, de la casa "BioEco", este producto es un descomponedor y el efecto que se buscaba con el empleo de este producto era la descomposición del rastrojo de arroz y de caña principalmente, que causan gran heterogeneidad en la densidad de siembra del arroz (Bio eco 2017); b) Trichoderma que es un fungicida orgánico compuesto por una mezcla de Trichoderma harzianum, T. asperellum, aislado y desarrollado por el Laboratorio de microbiología de Suelos de la Universidad de Costa Rica para combatir principalmente problemas causados en los cultivos por hongos como Sclerotium spp., Phytophthora parasítica, Pythium spp., Phytophthora cinamomi, Alternaria spp., Thielaviopsis paradoxa, Fusarium spp., Rhizoctonia solani, Aspergillus spp., Mycosphaerella citri, Botrytis cinérea, Cladosporium spp., Colletotrichum spp., Rhizopus spp (Centro de Investigaciones Agronómicas 2015); c) Nitrobacter es una alternativa a la fertilización nitrogenada por medio de químicos, en ese caso se utiliza lo que popularmente se conoce como "solubilizadores de nitrógeno" "que puede ayudar a disminuir el uso de fertilizantes sintéticos tradicionales, mediante el uso de bacterias capaces de fijar nitrógeno en suelos agrícolas, disminuyendo la posibilidad de contaminación de aguas subterráneas, ríos, lagos; que causan daños a la salud humana y animal" (Villegas 2016); d) Bioactivado ${ }^{\circledR}$ catalogado como acondicionador de suelo; es una mezcla de Azotobacter sp., Bacillus subtilis, Lactobacillus sp., Actinomycetes: Streptomyces sp y Sacharomyces . Su función es mejorar la sanidad del cultivo y enriquecer la microbiota del suelo. Aumenta las poblaciones de microorganismos benéficos nativos y mejora el aprovechamiento de la fertilización química; acompleja las moléculas de fertilizantes a base de sales (urea, sulfato de magnesio, etc), aumentando su absorción. Solubiliza nutrientes en el suelo, separando las moléculas que los mantienen fijos como el calcio y fósforo, contribuyendo así a su absorción por las raíces. Controla bacterias dañinas a nivel de suelo.

Su contenido de Nitrógeno es aportado por fuentes microbiales como Azotobacter. Las bacterias del género Azotobacter de las cuales está compuesto el Bioactivado, poseen la habilidad de convertir el dinitrógeno atmosférico (N2) a amonio (NH4+) mediante la acción de la enzima nitrogenasa. Producen vitaminas y sustancias estimuladoras del crecimiento (ácido indolacético, ácido, giberélico, ácido nicotínico, ácido pantoténico, citoquininas y vitaminas como biotina) que influyen directamente en el desarrollo vegetal. Posee el 
producto la capacidad de solubilizar fosfatos. Modifica la flora del suelo, evitando la colonización de patógenos por competencia directa de nutrimentos y espacio. Limita el crecimiento de patógenos ya que consume la infección y tejidos muertos que estos generan. Genera sustancias antimicrobianas que eliminan al ente patógeno. Suprime microorganismos causantes de enfermedades Fusarium, Erwiniay Xantomonas. Los Streptomyces producen metabolitos con función antibiótica e inmunosupresora para el combate de enfermedades causadas por hongos y bacterias como: Erythromicina, Neomycina, Streptomicina, Gentamicina, entre otros. Bioactivado contiene bacterias y bacillus que producen acido láctico el cual es un fuerte esterilizador que suprime microorganismos patógenos (Cruz 2015).

Para la realización de esta investigación se recurrió al modelo de Cuasi-experimento (Bono 2012) ya que la logística del ensayo no permitía una aleatorización ortodoxa dado que la dinámica de los microorganismos en un modelo tipo Irrestricto hubiera exigido la confección de una gran cantidad de canales que aislaran las unidades experimentales para evitar la contaminación entre tratamientos, por esta razón se seleccionó cuatro bandas de $14 \mathrm{~m}$ de ancho por 50 de largo separadas por un canal suficientemente ancho para aislar completamente las bandas; posteriormente la colocación de los microorganismos se realizó aleatoriamente en las bandas y en cada banda se estableció una cuadrícula de $10 \mathrm{~m} \mathrm{X} 10 \mathrm{~m}$ para un total de 100 parcelas básicas que componen - dentro de cada banda - un ensayo blanco que permitiera posteriormente establecer el tamaño óptimo de unidad experimental y la variabilidad del suelo según el índice de Smith.

Resultados

En el Tabla 1 se muestran los índices de heterogeneidad establecidos por el procedimiento de Smith (1938) (Gráfico 2); en todos los casos el valor que se presenta es el obtenido por medio de la ponderación por grados de libertad propuesta por Federer (1963) dado que las pendientes de las regresiones que se muestran en el gráfico 2 todas son mayores que 1 y el índice de Smith oscila entre 0 y 1 , donde el cero es completa homogeneidad y el 1 es completa heterogeneidad. 
Tabla 1 : Índice de heterogeneidad del suelo medido en las parcelas donde se evaluó cada uno de los productos. Bagaces, Guanacaste, Cosa Rica. 2014

\begin{tabular}{lr}
\hline \multicolumn{1}{c}{ Producto } & $\begin{array}{c}\text { Índice de heterogeneidad } \\
\text { de Smith }\end{array}$ \\
\hline Bioactivado & 0,33 \\
Micro Plus & 0,51 \\
NitroBacter & 0,66 \\
Trichoderma & 0,60 \\
\hline
\end{tabular}
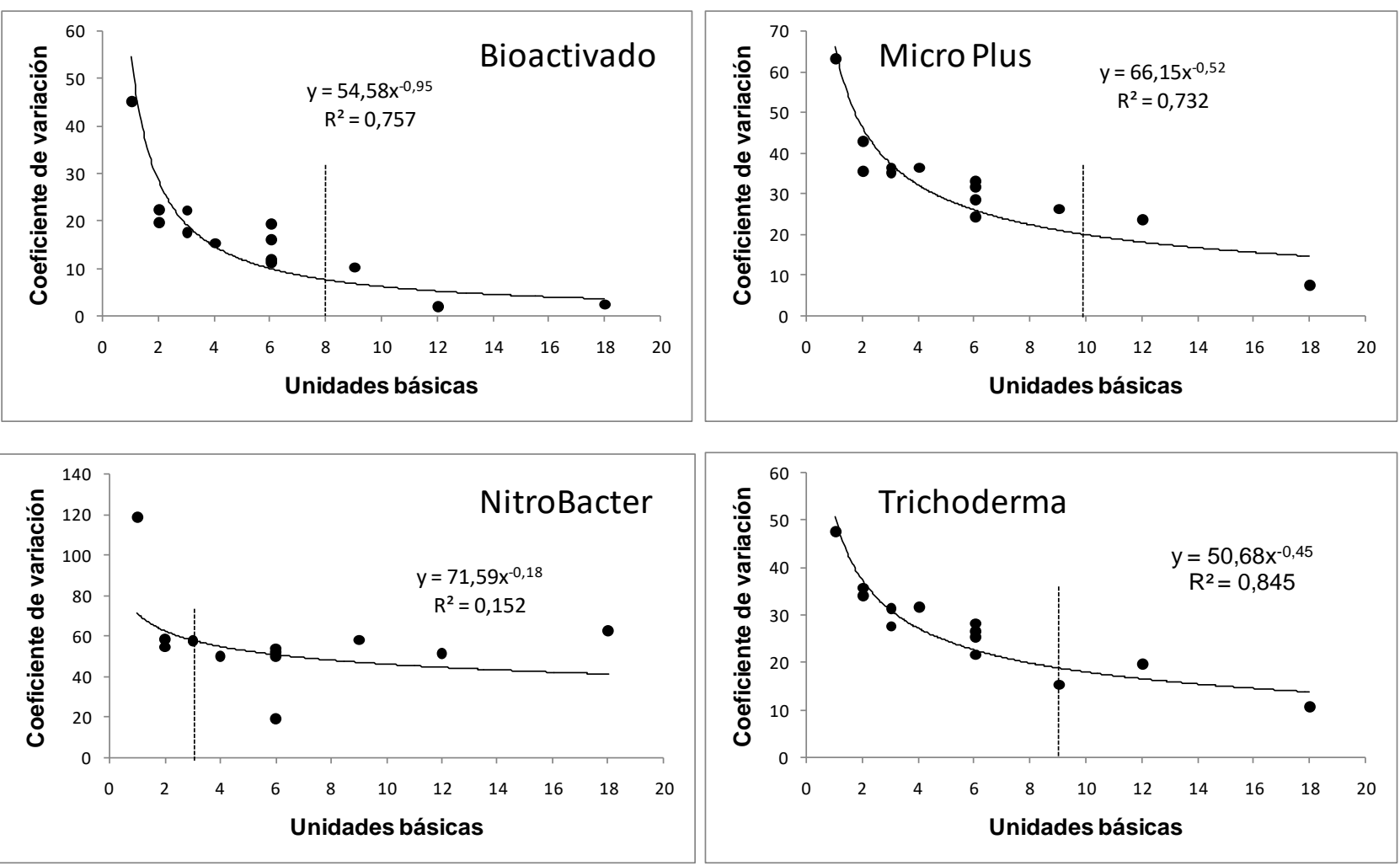

Gráfico 1: Relación entre unidades básicas y Coeficientes de variación para los cuatro productos a base de microorganismos evaluados en los ensayos de uniformidad en Bagaces, Guanacaste, Costa Rica 2014 
Bioactoivado

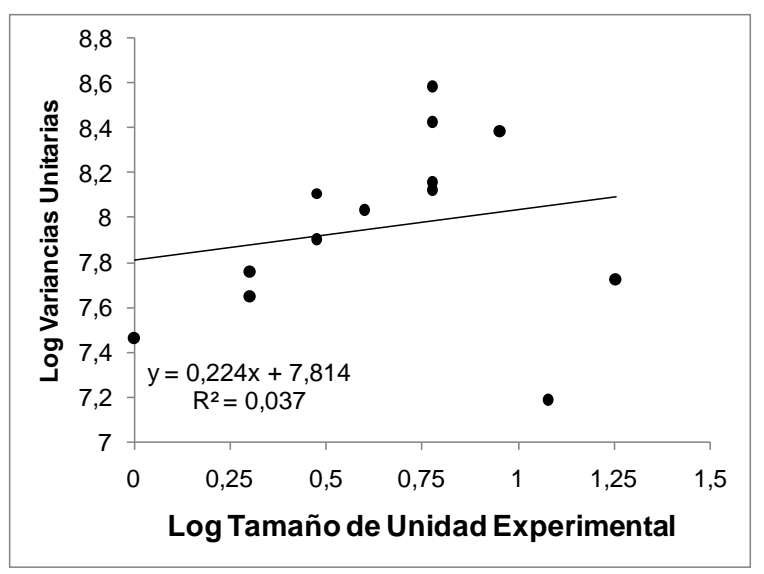

Nitrobacter

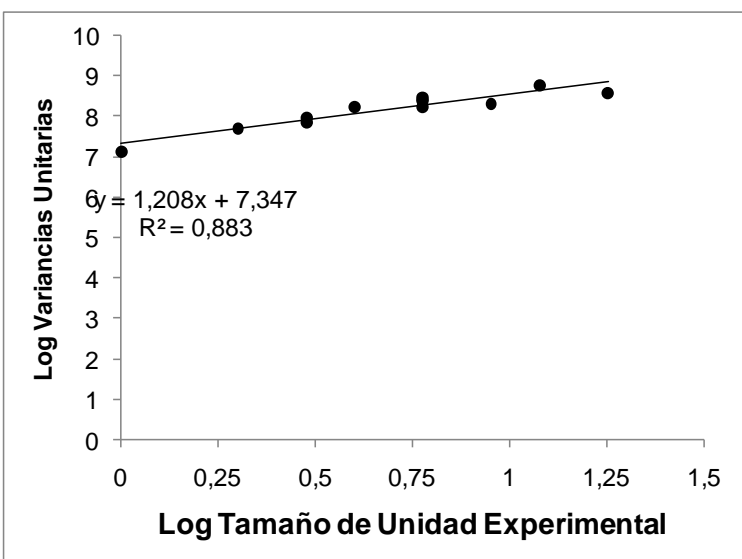

Micro Plus

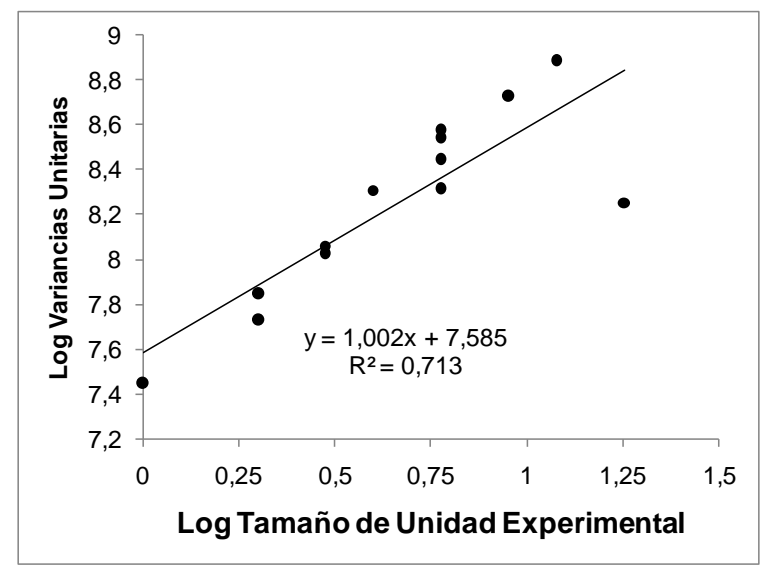

Trichoderma

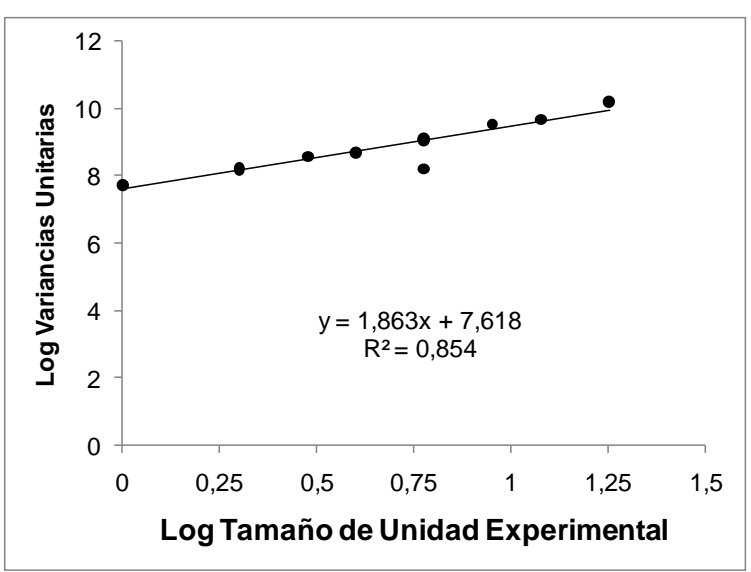

Gráfico 2:Regresiones para el cálculo del índice de Smith (pendiente de la ecuación de regresión) para los ensayos de uniformidad en Bagaces, Guanacaste, Costa Rica. 2014 

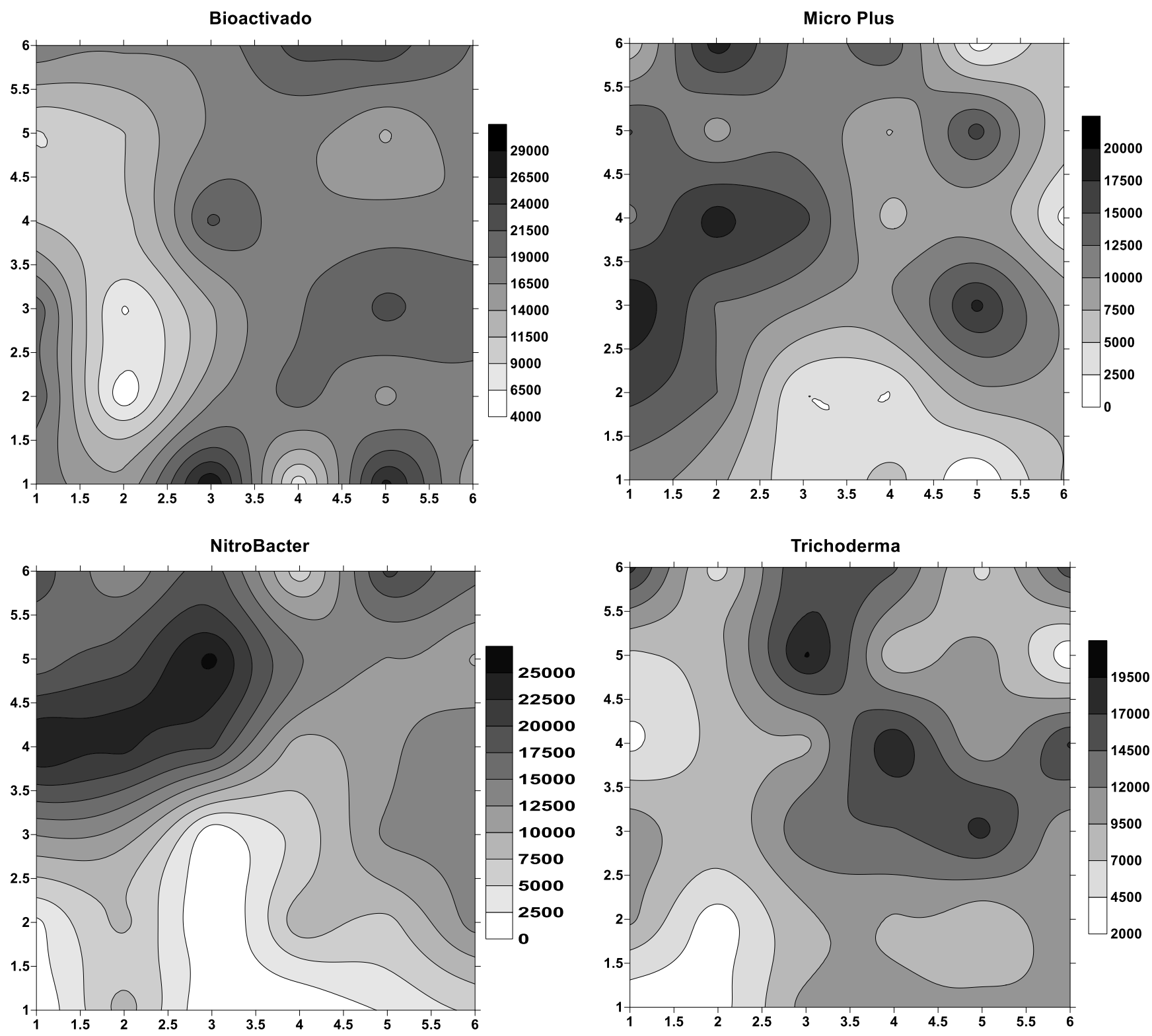

Gráfico 3: Representación gráfica de la heterogeneidad del suelo de los sitios en que se probó cada uno de los productos a base de microorganismos en La Hacienda Mojica, Bagaces, Guanacaste, Costa Rica. 2014.

En el gráfico 3 se muestra los mapas que representas gráficamente la heterogeneidad del suelo por medio de modelos matemáticos llamados semivariancia e interpolaciones obtenidas mediante un proceso denominando "kriging" el cual nunca fue traducido al español; esto se hace con el fin de obtener un conjunto denso de puntos para que los mapas queden mejor definidos.

Es sencillo correlacionar visualmente los valores de heterogeneidad de Smith con los mapas del gráfico 2, por ejemplo, el mapa del producto Bioactivado es el más sencillo de los cuatro mapas 
y a la vez es el índice de Smith (Tabla 1) más pequeño. También se realizó un análisis de variancia para comparar los diferentes productos; el resultado de dicho análisis se presenta en el Tabla 2.

Tabla 2: Prueba DGC para los distintos tratamientos microorganismos. Bagaces, Guanacaste, Costa Rica. 2014

\begin{tabular}{lrrrcc}
\hline \multicolumn{1}{c}{ tratamiento } & \multicolumn{1}{c}{ Medias } & $\mathrm{n}$ & \multicolumn{3}{c}{ Clasificación } \\
\hline Micro Plus & 9802,78 & & 36 & $\mathrm{~A}^{*}$ & \\
Trichoderma & 10344,44 & & 36 & $\mathrm{~A}$ & \\
Nitrobacter & 11316,67 & 36 & $\mathrm{~A}$ & \\
Bioactivado & 16880,56 & 36 & & $\mathrm{~B}$ \\
\hline
\end{tabular}

*Medias con una letra común no son significativamente diferentes $(p>0,05)$

La variable producción es por naturaleza normal por lo que sólo se realizó la prueba de Levene para la homogeneidad de variancias que probó que no existe heterocedasticidad en los datos. Posteriormente el análisis de variancia mostró que había diferencia entre productos por lo que se realizó una prueba de separación de medias DGC.

\section{Discusión}

El objetivo principal de este trabajo era demostrar si la adición de poblaciones de microorganismos diferentes podría provocar una dinámica más vigorosa en el suelo lo que podría reflejarse en un aumento en la heterogeneidad del mismo y por tanto un aumento en el tamaño óptimo de la Unidad Experimental estimado anteriormente (Vargas y Navarro 2014). Es obvio con los resultados obtenidos en este trabajo que el uso de productos a base de microorganismos no produce aumento en la heterogeneidad del suelo por lo que el tamaño recomendado en el trabajo de Vargas y Navarro (2014) de $20 \mathrm{~m}^{2}$ se mantiene vigente hasta tanto no se verifique un cambio radical de las prácticas del cultivo del arroz en la zona.

Los índices de heterogeneidad obtenidos en este ensayo fueron bastante más bajos que el obtenido por Vargas y Navarro (2014) de 0,83 lo que indica que en una misma finca se pueden hallar suelos de muy diversa naturaleza debido a la génesis del piemonte de la cordillera volcánica de Guanacaste en donde confluyeron gran cantidad de coladas de lava de los varios volcanes cercanos y además de la actividad aluvial de los ríos presentes en la zona, lo que dio como resultado un mosaico de suelos que se refleja en los índices que se han encontrado en los ensayos realizados en la zona. 
Es importante la recomendación de Vargas y Navarro (2014) de que primero se debe conocer el índice de heterogeneidad del suelo antes de tomar la decisión sobre el tamaño de la unidad experimental a emplear en un ensayo por lo que el autor no recomienda un número exacto sino que un ámbito entre $15 \mathrm{~m}^{2}$ y $20 \mathrm{~m}^{2}$, aunque podría ser más pequeña en suelos mucho más homogéneos como los que se encontraron en este trabajo.

El empleo de productos a base de microorganismos demuestra ser benéfico no solo por la adición de microfauna y microflora al suelo sino porque también se refleja en la producción de grano. En este trabajo, el producto denominando Bioactivado rindió una producción de 17 tm/ha lo que triplica el promedio normal de la finca. Si bien es cierto este es un resultado experimental el producto se muestra promisorio para la producción comercial del grano.

\section{Referencias bibliográficas:}

Alvarez, R.; Soto, M.; Gómez, H. 1986. Tamaño de parcela y número de repeticiones para estimar una población de Spodoptera frugiperda (Smith) y su daño en maíz. Revista Facultad Nacional de Agronomía 39(2): 6-16.

Barrientos García, M. 1981. Evaluación de 4 métodos para la determinación de tamaño y forma óptimos de parcela para experimentación agrícola. Tesis Licenciatura. San Carlos, Guatemala. Universidad de San Carlos. 79 p.

Bioeco. 2017. Micro Plus: producto concentrado y formulado en medio de cultivos bacterianos, a base de bacterias benéficas para control de malos olores y descomposición de desechos orgánicos. http://www.greensolutionscr.com/microplus-bacterias-descomponedoras

Bolaños, M.; Watson, V.;Tosi, O. 2005. Mapa ecológico de Costa Rica (zonas de vida) según el sistema de clasificación de zonas de vida del mundo de L.R. Holdrige. Centro Científico Tropical, San José, Costa Rica. Mapa.

Bono, R. 2012. Diseños cuasi-experimentales y longitudinales. Departamento de metodología de las ciencias del comportamiento, Facultad de Psicología, Universidad de Barcelona. 86 pp.

Box, G.; Stuart, J.; Hunter, W. 2008. Estadística para investigadores: diseño, innovación y descubrimiento. Editorial Reverté, Barcelona, España. 639 p.

Chandler, R. 1984. Arroz en los trópicos: guía para el desarrollo de programas nacionales. IICA, Costa Rica. 304 p.

Cruz, Y. 2015. Uso de enmiendas biológicas (enzimas, hongos benéficos y microorga-nismos eficientes) en la instalación del sistema "Vetiver" en taludes de corte con baja fertilidad. 
The Vetiver Network Inernational, VI conferencia internacional del Vetiver (ICV) Danang, Vietnam, Mayo 20159 pp. http://www.vetiver.org/ICV6_PROC/BIOENGINEERING\%20/13\%20Y\%20CRUZ\%20 Spanish.pdf

Custódio da Silva, W. 2010. Comparacao de métodos para estimar o tamaño ótimo de parcela em bananeira. Tesis Mag.Sc. Vicosa, Brazil. Universidad Federal de Vicosa. 37 p.

Escobar, C. 1981. Estimación del tamaño óptimo de parcela experimental para ensayos de rendimiento con maíz. Revista Facultad Nacional de Agronomía Medellín (34)1:31-36

Federer, W.T. 1963. Experimental design. New York. MacMillian. 544 p.

Gomez, K.; Gomez, A. 1984. Statistical procedures for agricultural research. 2da ed. John Wiley \& Sons, Philippines. 680 p.

Kavitha, B. 2010. Study on optimum plot size and optimum plot shape of soybean crop. Tesis Mag.Sc. Dharwad, India. University of Agricultural Sciences. 58 p.

Primuslabs. 2016. Lista de insumos aprobados por Primuslabs para su uso en agricultiura orgánica. http://www.primuslabs.com/docs/lista\%20de\%20insumos\%20evaluados.pdf

Rodríguez, N.; Sánchez, H.; Pacheco, P. 1993. Determinación de tamaño y forma óptimos de parcela para ensayos de rendimiento con café. Revista Colombiana de Estadística. 14(27): 50-64.

Rosselló, J.M.; Fernández, M. 1986. Guía técnica para ensayos de variedades de campo. FAO. 144 p.

Smith, F. 1938. An empirical law describing heterogeneity in the yields of agricultural crops. Journal of Agriculture Science 28:1-23.

Vargas, J.C.; Navarro, J.R. 2014. Determinación de un tamaño adecuado de unidad experimental, utilizando el método de curvatura máxima, para ensayos de arroz (Oryza sativa), en Bagaces Guanacaste. Intersedes Vol. 15 Núm. 31: 128 - 144

Villegas, 1. 2016. Efecto de la aplicación del producto Nitrobacter ${ }^{\circledR}$ dentro de un plan de fertilización nitrogenada sobre el crecimiento y rendimiento de melón dorado (Ciucumis melo L.) híbrido 6276 en Pilas de Canjel, Nandayure, Guanacaste. Tesis Ingeniero Agrónomo, Instituto Tecnológico e Costa Rica, San Carlos, Alajuela, Costa Rica. 65 pp. 